\begin{tabular}{|c|l|}
\hline Title & Static stability of the Jovian atmospheres estimated from moist adiabatic profiles \\
\hline Author(s) & Sugiyama, Ko-ichiro; Odaka, Masatsugu; Kuramoto, Kiy oshi; Hay ashi, Y oshi-Y uki \\
\hline Citation & $\begin{array}{l}\text { Geophysical Research Letters, 33(3), L03201 } \\
\text { https://doi.org/L0.1029/2005GLO24554 }\end{array}$ \\
\hline Issue Date & 2006-02-01 \\
\hline Doc URL & http://hdl.handle.net/2115/8293 \\
\hline Type & article(author version) \\
\hline Note & An edited version of this paper was published by A GU. Copyright 2006 A merican Geophysical Union. \\
\hline File Information & Sugiyama etal_huscap.pdf \\
\hline
\end{tabular}

Instructions for use 


\title{
Static stability of the Jovian atmospheres estimated from moist adiabatic profiles
}

\author{
Ko-ichiro Sugiyama, Masatsugu Odaka, Kiyoshi Kuramoto, \\ and Yoshi-Yuki Hayashi \\ Division of Earth and Planetary Sciences, Hokkaido University, Sapporo, Japan
}

The dependency of static stability $N^{2}$ of the Jovian atmospheres on the abundances of condensible elements is considered by calculating the moist adiabatic profiles. An optimal minimization method of the Gibbs free energy is utilized to obtain equilibrium compositions in order to cover a variety of basic elements. It is shown that $\mathrm{CH}_{4}$ is one of the dominant contributors to producing a stable layer in the Uranian atmosphere. On Jupiter, Achterberg and Ingersoll [1989] have shown that, at low water abundances, $N^{2}$ is proportional to the $\mathrm{H}_{2} \mathrm{O}$ abundance. In the present study, we show that this relationship does not hold when the $\mathrm{H}_{2} \mathrm{O}$ abundance is larger than approximately $5 \times$ solar. A rough estimation of wave speed indicates that the abundance of $10 \times$ solar is marginal to explain the SL9-induced wave speed as that of an internal gravity wave.

\section{Introduction}

Static stability $N^{2}$ (or buoyancy frequency $N$ ) is one of the basic parameters in considering the dynamics of stratified atmospheres. Achterberg and Ingersoll [1989] estimate the maximum value of $N^{2}$ and its relationship with deep water abundance in the Jovian atmosphere. They assume that the temperature and composition of the cloud layer follow moist adiabatic profiles and that $N^{2}$ can be evaluated from the difference between moist and dry adiabats. They conclude that the maximum value of $N^{2}$ is proportional to the $\mathrm{H}_{2} \mathrm{O}$ mole fraction $x_{\mathrm{H}_{2} \mathrm{O}}$ of a deep atmosphere in the range from $x_{\mathrm{H}_{2} \mathrm{O}}=1.0 \times 10^{-4}$ to $5.0 \times 10^{-3}$. Assuming this linear relationship, Ingersoll and Kanamori [1995] argue that the phase velocity of the SL9-induced wave can be explained as that of an internal gravity wave trapped in the stable layer if $x_{\mathrm{H}_{2} \mathrm{O}}$ is approximately $1.5 \times 10^{-2}$. This is the abundance of $\mathrm{H}_{2} \mathrm{O}$, which can be obtained when the abundance of $\mathrm{O}$ is approximately $10 \times$ solar $^{\dagger 1}$.

As for the Jovian atmospheres, there are multiple condensible volatiles and chemical processes. This poses some difficulty in the parametric study of $N^{2}$ for these atmospheres. Previous studies, such as Weidenschilling and Lewis [1973], Atreya and Romani [1985], and Achterberg and Ingersoll [1989], calculated the equilibrium composition of the atmosphere from the Clausius-Clapeyron equation. For the usage of the Clausius-Clapeyron equation, it is necessary to specify the set of independent chemical reactions that occur in the atmosphere considered. However, the specification of these reactions is generally not so easy.

In order to obtain the equilibrium composition of the atmosphere, we do not have to specify the set of chemical

Copyright 2006 by the American Geophysical Union. 0094-8276/06/\$5.00 reactions. The equilibrium composition can also be determined by minimizing the Gibbs free energy. In the present study, we develop a method for calculating moist adiabatic profiles by minimizing the Gibbs free energy, which enables us to cover a wider variety of compositions, compared to previous studies. In the following, the dependency of $N^{2}$ on the abundances of condensible elements in the atmospheres of Jupiter and Uranus is examined.

\section{Calculation Method}

\subsection{Gibbs free energy and equilibrium composition}

We consider herein an atmosphere in thermodynamic equilibrium. When pressure and temperature are given, the equilibrium composition is calculated by minimizing the Gibbs free energy, $G$, under the condition that the total number of each element is conserved. Assuming an ideal gas and an ideal solution, $G$ can be expressed as

$$
\begin{aligned}
& G(T, p)=\sum_{i} \sum_{\phi} n_{i}^{\phi} \mu_{i}^{\phi}\left(T, p, n_{i}^{\phi}\right), \\
& \mu_{i}^{\phi}\left(T, p, n_{i}^{\phi}\right)=\mu_{i}^{\circ \phi}(T)+R T \ln x_{i}^{\phi}+\delta_{1 \phi} R T \ln p / p_{0},
\end{aligned}
$$

where $T$ is the temperature, $p$ and $p_{0}$ are the pressure and standard pressure, $n_{i}^{\phi}$ and $x_{i}^{\phi} \equiv n_{i}^{\phi} / \sum_{i} n_{i}^{\phi}$ are the mole number and mole fraction, respectively, of chemical species $i$ in phase $\phi, \mu_{i}^{\phi}$ and $\mu_{i}{ }^{\circ \phi}$ are the chemical potential and chemical potential at the standard pressure, respectively, and $R$ is the gas constant [see also Sugiyama et al., 2001]. Here, the gas phase is indicated by $\phi=1 . \delta_{1 \phi}$ is the Kronecker delta. We assume that only the chemical potential of the gas phase depends on pressure.

The chemical potential of each gas species at the standard pressure is calculated from the following equation:

$$
\begin{aligned}
\mu_{i}{ }^{\circ \phi}(T)= & h_{i}^{\phi}\left(T_{0}\right)-T s_{i}^{\phi}\left(T_{0}, p_{0}\right) \\
& +\int_{T_{0}}^{T} c_{p_{i}}^{\phi}(T) d T-T \int_{T_{0}}^{T} \frac{c_{p_{i}}^{\phi}(T)}{T} d T, \quad \text { for } \phi=1,
\end{aligned}
$$

where $T_{0}$ is the standard temperature, $h_{i}^{\phi}$ is the molecular enthalpy, $s_{i}^{\phi}$ is the molecular entropy, and $c_{p_{i}}^{\phi}$ is the specific heat at constant pressure. The values of $h_{i}^{\phi}\left(T_{0}\right), s_{i}^{\phi}\left(T_{0}, p_{0}\right)$, and $c_{p}{ }_{i}^{\phi}(T)$ are adopted from NIST-JANAF Thermochemical Tables [1989]. Spline interpolation is used to give a functional form of $c_{p_{i}}{ }^{\phi}(T)$. The chemical potential of each condensed species at the standard pressure, except for $\mathrm{NH}_{4} \mathrm{SH}$, is given by

$\mu_{i}^{\circ \phi}(T)=\mu_{i}^{\circ 1}(T)+R T \ln e_{i}(T) / p_{0}, \quad$ for $\phi \neq 1$,

where $e_{i}(T)$ is the saturated vapor pressure of species $i$, which is evaluated by the Antoine equation [Chemical Handbook, 1993]. The values of Antoine coefficients are adopted 
from the Chemical Handbook [1993]. In the lower temperature range, where the Antoine equation is not valid, $\mu_{i}{ }^{\circ \phi}(T)$ is given as a quadratic function of temperature that is smoothly connected to the above expression. The chemical potential of the condensed phase of $\mathrm{NH}_{4} \mathrm{SH}$ at the standard pressure is calculated from the equilibrium constant for $\mathrm{NH}_{4} \mathrm{SH}$ formation reaction [Lewis, 1969].

The RAND method [White et al., 1953; Van Zeggeren and Storey, 1970, Wood and Hashimoto, 1993] is used to obtain equilibrium composition. During the application of the RAND method, we examine whether or not each condensed phase really equilibrates with the atmospheric gas phase under the given temperature and pressure. This check of phase stability is required in order to ensure non-singularity to the coefficient matrix of the RAND method, to avoid an optimized solution converging to local minimum, and to accelerate the execution speed of numerical calculation of the RAND method. Our source code is available at http://www.gfd-dennou.org/library/oboro/.

\subsection{Moist adiabatic profile}

The vertical profiles of temperature, composition, and condensates are obtained by considering an air parcel following the pseudo moist adiabatic process. We assume that all of the condensates are removed from the air parcel, while the value of total entropy, which is the sum of those for gas and already removed condensates at each pressure level, is conserved.

\subsection{Static stability}

The value of $N^{2}$ is estimated as described by Achterberg and Ingersoll [1989]. The profiles of the temperature and mean molecular weight are those obtained for the pseudo moist adiabatic process. The value of $N^{2}$ is then given as follows:

$N^{2}=\frac{g}{T}\left(\frac{d T}{d z}+\frac{M g}{c_{p}}\right)-\frac{g}{M} \frac{d M}{d z}$,

where $g$ is the acceleration of gravity, $M$ is the mean molecular weight, and $c_{p}$ is the mean specific heat per mole. The interpretation of the moist adiabat as a rough proxy of the mean atmospheric thermal structure is based on the knowledge of the earth's troposphere (e.g., Gill [1982]). Although dynamics may alter the detailed profiles of $T$ and $M$, as is exemplified by Nakajima et al. [2000], we believe that this formula is still applicable to arguments concerning the order of magnitude.

The $N^{2}$ given by equation (1) is slightly different from that of Achterberg and Ingersoll [1989] with respect to the definition of specific heat. We herein adopt the mean specific heat of an air parcel at a given pressure level, whereas Achterberg and Ingersoll [1989] adopt that of a condensiblefree air parcel. They assume that the abundances of any condensible elements in the Jovian atmospheres are sufficiently small. Our value of $N^{2}$ is approximately the same as that of Achterberg and Ingersoll [1989] in the parameter ranges considered in their study.

\section{Results for the Atmospheres of Jupiter and Uranus}

The equilibrium profiles of the Jovian and Uranian atmospheres are calculated to pass through the following temperature and pressure points that are determined from observations: $150 \mathrm{~K}$ at $6 \times 10^{4} \mathrm{~Pa}$ adopted from Eshleman et al. [1979] for Jupiter, and $101 \mathrm{~K}$ at $2.3 \times 10^{5} \mathrm{~Pa}$ from Lindal et al. [1987] for Uranus. All of the components considered in our calculations are listed in Table $1 . \mathrm{H}_{2} \mathrm{O}(\mathrm{l}), \mathrm{NH}_{3}(\mathrm{l})$, and $\mathrm{H}_{2} \mathrm{~S}(\mathrm{l})$ are assumed to form an ideal solution, while $\mathrm{CH}_{4}(\mathrm{l})$ is isolated. Any combination of $\mathrm{H}_{2} \mathrm{O}(\mathrm{s}), \mathrm{NH}_{3}(\mathrm{~s}), \mathrm{H}_{2} \mathrm{~S}(\mathrm{~s})$, $\mathrm{CH}_{4}(\mathrm{~s})$, and $\mathrm{NH}_{4} \mathrm{SH}(\mathrm{s})$ is assumed not to be a solid solution. The ratio of ortho- to para-hydrogen is assumed to be that of the equilibrium value. The abundances of non-condensible elements such as $\mathrm{H}$ and $\mathrm{He}$ are taken at the solar abundance, and the abundances of condensible elements, such as $\mathrm{C}, \mathrm{N}$, $\mathrm{O}$, and $\mathrm{S}$, are varied from $1 \times$ solar to $50 \times$ solar abundance. This range is given considering that the Uranian atmosphere may be rich in $\mathrm{C}$ with $30 \times$ solar $^{\dagger}$ [Bergstralh and Miner, 1991] and also in S with $10 \times \operatorname{solar}^{\dagger}$ [de Pater et al., 1991]. The solar abundance adopted in the present study is that revised by Asplund et al. [2005], whereas that of Anders and Grevesse [1989] is used in the previous studies cited above. Each elemental abundance of He, C, N, O, and S is reduced from that of Anders and Grevesse [1989] by 13\%, 33\%, 46\%, $46 \%$, and $15 \%$, respectively.

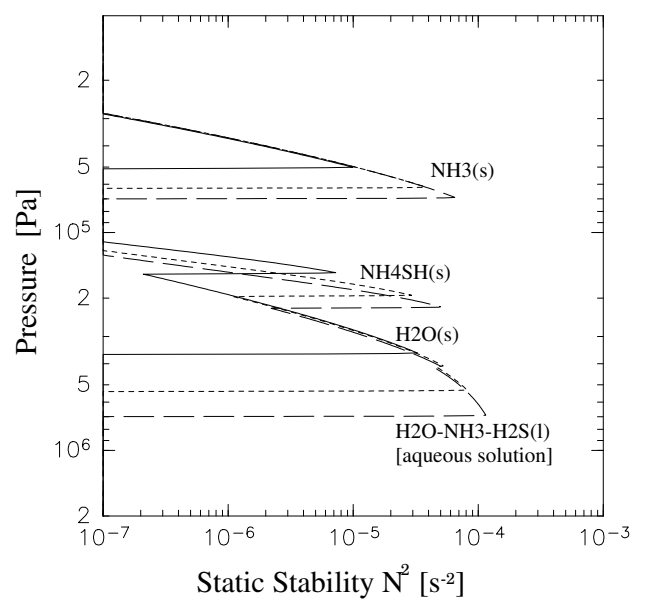

Figure 1. Profiles of $N^{2}$ of the Jovian atmosphere. The abundance of condensible volatiles are taken at $1 \times$ solar (solid line), $5 \times$ solar (dotted line), and $10 \times$ solar (dashed line), respectively.

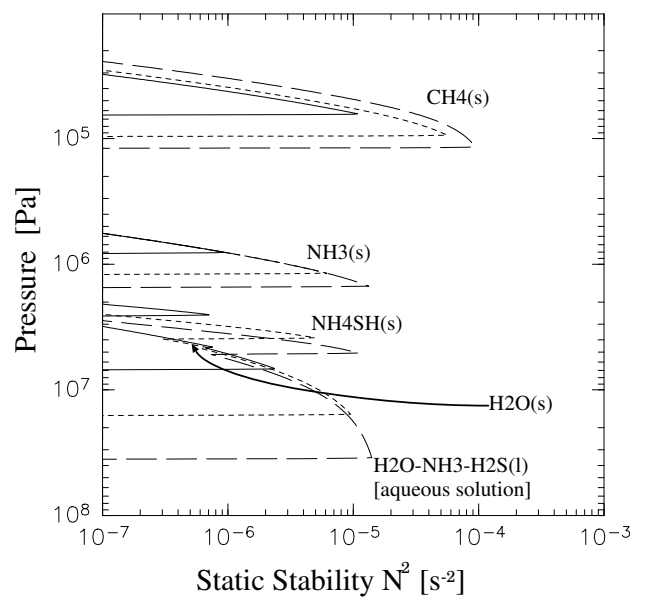

Figure 2. Profiles of $N^{2}$ of the Uranian atmosphere. The abundance of condensible volatiles are taken at $1 \times$ solar (solid line), $10 \times$ solar (dotted line), and $30 \times$ solar (dashed line), respectively. 
Table 1. Components considered in our model atmosphere.

\begin{tabular}{lll}
\hline gas (g) & liquid (l) & solid (s) \\
\hline $\mathrm{H}_{2}, \mathrm{He}, \mathrm{H}_{2} \mathrm{O}$, & $\mathrm{H}_{2} \mathrm{O}, \mathrm{NH}_{3}$, & $\mathrm{H}_{2} \mathrm{O}, \mathrm{NH}_{3}, \mathrm{H}_{2} \mathrm{~S}$, \\
$\mathrm{CH}_{4}, \mathrm{NH}_{3}, \mathrm{H}_{2} \mathrm{~S}$ & $\mathrm{H}_{2} \mathrm{~S}, \mathrm{CH}_{4}$ & $\mathrm{CH}_{4}, \mathrm{NH}_{4} \mathrm{SH}$ \\
\hline
\end{tabular}

Figure 1 shows the vertical profiles of $N^{2}$ for the Jovian atmosphere calculated for the cases of condensible elements at 1,5 , and $10 \times$ solar abundance. Condensed species are the same as those obtained by Atreya and Romani [1985]. $\mathrm{H}_{2} \mathrm{O}(\mathrm{s}), \mathrm{NH}_{4} \mathrm{SH}(\mathrm{s})$, and $\mathrm{NH}_{3}(\mathrm{~s})$ condense for all cases, and $\mathrm{H}_{2} \mathrm{O}-\mathrm{NH}_{3}-\mathrm{H}_{2} \mathrm{~S}(\mathrm{l})$ condenses for the cases of 5 and $10 \times$ solar. The profiles of $N^{2}$ have many peaks that correspond to lifting condensation levels of condensible species. The lifting condensation level of each condensed species is higher than that obtained by Atreya and Romani [1985], because the solar abundance is reduced compared to that used in previous studies. The most stable layer in the Jovian atmosphere is produced by $\mathrm{H}_{2} \mathrm{O}$ condensation. The maximum value of $N^{2}$ associated with $\mathrm{H}_{2} \mathrm{O}$ condensation is several times larger than that of $\mathrm{NH}_{3}$. In addition, the thickness of the layer corresponding to $\mathrm{H}_{2} \mathrm{O}$ condensation is roughly three times larger than the other layers.

The maximum values of $N^{2}$ for the $1 \times$ solar case are smaller than those obtained by Achterberg and Ingersoll [1989], because of the solar abundance of Asplund et al. [2005]. These values increase with the increase in the abundances of condensible elements. However, it is noteworthy that the maximum value of $N^{2}$ caused by $\mathrm{H}_{2} \mathrm{O}$ condensation does not increase greatly with the increase in the abundances of condensible elements. This point will be discussed in detail in the next section.

Figure 2 shows the vertical profiles of $N^{2}$ for the Uranian atmosphere calculated for the cases of condensible elements at 1,10 , and $30 \times$ solar. $\mathrm{H}_{2} \mathrm{O}-\mathrm{NH}_{3}-\mathrm{H}_{2} \mathrm{~S}(\mathrm{l}), \mathrm{H}_{2} \mathrm{O}(\mathrm{s})$, $\mathrm{NH}_{4} \mathrm{SH}(\mathrm{s})$, and $\mathrm{NH}_{3}(\mathrm{~s})$ condense for all cases. The nonideal gas effect, which is neglected here, is estimated using the formula of Reid et al. [1987] to be approximately 10\% at the $\mathrm{H}_{2} \mathrm{O}$ solution cloud base level. Note that the $\mathrm{CH}_{4}(\mathrm{~s})$ curves in Figure 2 do not lie on top of each other, because the reference temperature of our calculation is higher than

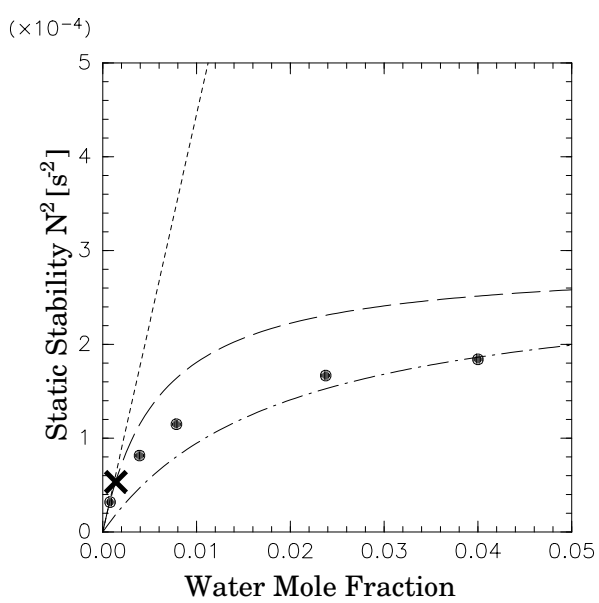

Figure 3. Maximum value of $N^{2}$ at the $\mathrm{H}_{2} \mathrm{O}$ ice or solution cloud base as a function of $x_{\mathrm{H}_{2} \mathrm{O}}$ of the Jovian atmosphere. Circles indicate those for 1, 5, 10, 30, and $50 \times$ solar abundance of Asplund et al. [2005]. Cross indicates that for $1 \times$ solar abundance of Anders and Grevesse [1989]. For descriptions of the lines, see the text. the $\mathrm{CH}_{4}$ condensation temperature. The maximum value of $N^{2}$ in the Uranian atmosphere is obtained for the $\mathrm{CH}_{4}$ condensation layer. When the abundance of each condensible element is the same, the maximum value of $N^{2}$ in the Uranian atmosphere is approximately $1 / 10$ that of the Jovian atmosphere. This is because $N^{2}$ is proportional to the square of the acceleration of gravity when the abundances of condensible elements are small (see equation (3)). The acceleration of gravity on the surface of Uranus is approximately $1 / 3$ that on the surface of Jupiter.

\section{Concluding Remarks}

A noteworthy point obtained from our results is that the dependency of the maximum value of $N^{2}$ on the deep water abundance is different from the linear relationship shown by Achterberg and Ingersoll [1989]. The linear relationship is confirmed for abundances of around $1 \times$ solar $^{\dagger}$, and is assumed by Ingersoll and Kanamori [1995] in considering the SL9-induced wave. Figure 3 indicates that the maximum value of $N^{2}$ does not increase linearly with the increase of $x_{\mathrm{H}_{2} \mathrm{O}}$. The assumption in earlier studies, in which the abundances of condensible elements are small, does not hold in the parameter range shown in Figure 3.

For simplicity, let us suppose that an atmosphere consists of two components: dry and condensible. Then, the expression of $N^{2}$ (equation (1)) can be written as

$$
\begin{aligned}
N^{2}= & \frac{g}{T}\left[\Gamma_{d}+\Gamma_{v}\left\{1-\frac{\left(M_{v}-M_{d}\right)}{M} \frac{\lambda_{v} x_{v}}{R T}\right\}\right] \\
& -\frac{g}{T} \frac{\left(M_{v}-M_{d}\right)}{M} \frac{M g x_{v}}{R},
\end{aligned}
$$

where $M_{d}$ and $M_{v}$ are the molecular weights of dry and condensible components, respectively, $x_{v}$ and $\lambda_{v}$ are the mole fraction and specific latent heat of condensible components, respectively, $c_{p_{d}}$ and $c_{p_{v}}$ are the specific heat of dry and condensible components, respectively, and $\Gamma_{d}=M g / c_{p}$ and $\Gamma_{v}=M g / c_{p}\left\{\left(1+\lambda_{v} x_{v} / R T\right) /\left(1+\lambda_{v}^{2} x_{v} / c_{p} R T^{2}\right)\right\}$ are the dry and moist adiabat lapse rates, respectively. In Figure 3, equation (2) is plotted as a function of $x_{\mathrm{H}_{2} \mathrm{O}}$ by dashed and dot-dashed curves. The dashed curve indicates the use of fixed values of $c_{p}, M, \lambda_{v}$, and $T$ that were obtained at the $\mathrm{H}_{2} \mathrm{O}$ ice cloud base for $1 \times$ solar, whereas the dot-dashed curve indicates the use of fixed values of $c_{p}, M, \lambda_{v}$, and $T$ that were obtained at the $\mathrm{H}_{2} \mathrm{O}$ solution cloud base for $50 \times$ solar. When the small abundance assumption is applicable, the above expression can be approximated by the following linear relationship:

$N^{2}=\frac{M_{d} g^{2}}{c_{p_{d}} T}\left(\frac{\lambda_{v}}{R T}-\frac{c_{p_{d}}}{R}\right)\left(\frac{\lambda_{v}}{c_{p_{d}} T}+\frac{M_{v}-M_{d}}{M_{d}}\right) x_{v}$.

The condition for equation (3) to be valid is $\lambda_{v}^{2} x_{v} / c_{p} R T^{2}$ $\ll 1$. Substitution of $T=300 \mathrm{~K}$ and $c_{p_{d}}=30 \mathrm{~J} \mathrm{~K}^{-1}$ $\mathrm{mol}^{-1}$ as typical values for Jupiter into this equation yields $x_{v} \ll 1 \times 10^{-2}$ for $\mathrm{H}_{2} \mathrm{O}$ with $\lambda_{v}=4.5 \times 10^{4} \mathrm{~J} \mathrm{~mol}^{-1}$. In Figure 3 , the dotted line represents equation (3) with fixed values of $c_{p_{d}}, \lambda_{v}$, and $T$ obtained at the $\mathrm{H}_{2} \mathrm{O}$ ice cloud base for $1 \times$ solar. The approximation given by equation (3) does not hold for Jupiter when $x_{\mathrm{H}_{2} \mathrm{O}}$ is larger than $3.9 \times 10^{-3}(5$ $\times$ solar).

Figure 3 indicates that the effect of the increase of the cloud base temperature is not negligible. As is expressed in equation (2), $N^{2}$ is proportional to $1 / T$. The cloud base temperature increases from $263 \mathrm{~K}$ at $1 \times$ solar to $371 \mathrm{~K}$ at $50 \times$ solar. Because of this temperature effect, the increase in the maximum value of $N^{2}$ even slows as $x_{\mathrm{H}_{2} \mathrm{O}}$ increases. 
An important implication of Figure 3 is that the maximum value of $N^{2}, 5 \times 10^{-4} \mathrm{~s}^{-2}$, which is required in the study by Ingersoll and Kanamori [1995] to explain the phase velocity of the SL9-induced wave, cannot be reached, even if the abundances of condensible elements are taken at 50 $\times$ solar. If the linear relationship holds, then $10 \times$ solar $^{\dagger}$ would be sufficient to explain the phase velocity. The beneficial point of the argument of Ingersoll and Kanamori [1995] was that $10 \times$ solar $^{\dagger}$ is within the acceptable range of other studies on the abundances of condensible elements.

Table 2. Estimated gravity wave speed of the Jovian stable layer corresponding to $\mathrm{H}_{2} \mathrm{O}$ condensation.

\begin{tabular}{llll}
\hline & $N^{2}\left[10^{-4} \mathrm{~s}^{-2}\right]^{\ddagger}$ & $H[\mathrm{~km}]^{\S}$ & $c[\mathrm{~m} / \mathrm{s}]\left(\int N d z\right)$ \\
\hline $1 \times$ solar & $0.31(0.6)$ & $40(0.93)$ & $96.8(0.74)$ \\
$1 \times$ solar & $0.52(1)$ & $43(1)$ & $130(1)$ \\
$10 \times$ solar & $1.15(2.2)$ & $63(1.47)$ & $370(2.85)$ \\
$15 \times$ solar & $1.35(2.60)$ & $70(1.62)$ & $475(3.66)$ \\
$20 \times$ solar & $1.49(2.87)$ & $75(1.74)$ & $568(4.37)$ \\
$30 \times$ solar & $1.67(3.2)$ & $85(1.98)$ & $731(5.63)$ \\
\hline$\ddagger$ Maximum values obtained at $\mathrm{H}_{2} \mathrm{O}$ ice or solution cloud base. \\
§ Thickness of layer where $N^{2}>1.0^{-7}$.
\end{tabular}

As mentioned by Ingersoll et al. [1994], the velocity of the gravity wave concerned is proportional not to the value of $N$, but rather to the integral of $N$ over the cloud layer thickness $H$. Table 2 summarizes the results of our calculation. Numbers in parentheses indicate the ratio of the corresponding values to $1 \times$ solar $^{\dagger}$, i.e., the solar abundance reported by Anders and Grevesse [1989]. The wave speed $c$ is assumed to be proportional to the vertical integral of $N$ over the stable layer composed of $\mathrm{H}_{2} \mathrm{O}-\mathrm{NH}_{3}-\mathrm{H}_{2} \mathrm{~S}(\mathrm{l}), \mathrm{H}_{2} \mathrm{O}(\mathrm{s})$, and $\mathrm{NH}_{4} \mathrm{SH}(\mathrm{s}) . \quad c=130 \mathrm{~m} / \mathrm{s}$ for the $1 \times$ solar $^{\dagger}$ case is adopted from Ingersoll and Kanamori [1995]. Table 2 indicates that the abundance of $10 \times$ solar is marginal to explain the SL9induced wave speed of $450 \mathrm{~m} / \mathrm{s}$. It may be worthwhile to recalculate the accurate gravity wave velocity using our profiles of $N^{2}$.

\section{Acknowledgments.}

The authors would like to thank A. Hashimoto for his lecture on the numerical method of minimizing the Gibbs free energy, K. Nakajima for his stimulating discussion concerning his numerical results for moist convection, and M. Ishiwatari and S. Watanabe for their useful comments. The authors would also like to thank the anonymous reviewer for helping us to improve our paper. This work was supported by a grant-in-aid (16204036) from the Japan Society for the Promotion of Science.

\section{Notes}

1. The solar abundance considered in Ingersoll and Kanamori [1995] is that reported by Anders and Grevesse [1989], not that reported by Asplund et al. [2005]. In the present study, "solar " " indicates the solar abundance reported by Anders and Grevesse [1989].

\section{References}

Achterberg, R. K., and A. P. Ingersoll (1989), A Normal-Mode Approach to Jovian Atmosphere Dynamics, J. Atmos. Sci., 46, 2448-2462.
Anders, E., and N. Grevesse (1989), Abundances of the elements: Meteoritic and solar, Geochim. Cosmochim. Acta, 53, 197-214.

Asplund, M., N. Grevesse, and A. J. Sauval (2005), The solar chemical composition, in Cosmic Abundances as Records of Stellar Evolution and Nucleosynthesis in honor of David L. Lambert, ASP Conference Series, 336, edited by T. G. Barnes and F. N. Bash., pp. 25-38 Astronomical Society of the Pacific, Scan Francisco.

Atreya, S. K., and P. N. Romani (1985), Photochemistry and clouds of Jupiter, Saturn and Uranus, in Recent Advances in Planetary Meteorology, edited by G. E. Hunt, pp. 17-68, Cambridge Univ. Press, London.

Bergstralh, J. T. and E. D. Miner (1991), The uranian system: an overview, In Uranus, edited by J. B. Bergstralh, E. D. Miner, and M. S. Matthews, pp. 3-25, The University of Arizona Press, Tucson.

Chase, M. W. (Eds.) (1989), NIST-JANAF Thermochemical Tables, 4th ed., AIP Press, New York.

de Pater, I., P. N. Romani, and S. K. Atreya (1991), Possible Microwave Absorption by $\mathrm{H}_{2} \mathrm{~S}$ Gas in Uranus' and Neptune's Atmosphere, Icarus, 91, 220-233.

Eshleman, V. R., G. L. Tayler, G. E. Wood, G. F. Lindal, J. D. Anderson, G. S. Levy, and T. A. Croft (1979), Radio science with Voyager 1 at Jupiter: preliminary profiles of the atmosphere and ionosphere, Science, 204, 976-978.

Gill, A. E., (1982), Atmosphere-Ocean Dynamics, Academic Press, SanDiego.

Ingersoll, A. P., H. Kanamori, and T. E. Dowling (1994), Atmospheric gravity waves from the impact of comet ShoemakerLevy 9 with Jupiter, Geophys. Res. Lett., 21, 1083-1086.

The Chemical Society of Japan (Eds.) (1993), Chemical Handbook (Kagaku-Binran), 4th ed., Maruzen, Tokyo, in Japanese.

Ingersoll, A. P., and H. Kanamori (1995), Wave from the collisions of comet Shoemaker-Levy 9 with Jupiter, Nature, 374 , 706-708.

Lewis, J. S. (1969), The Clouds of Jupiter and the $\mathrm{NH}_{3}-\mathrm{H}_{2} \mathrm{O}$ and $\mathrm{NH}_{3}-\mathrm{H}_{2} \mathrm{~S}$ Systems, Icarus, 10, 365-378.

Lindal, G.F., J. R. Lyons, D. N. Sweetnam, V. R. Eshleman, D. P. Hinson, and G. L. Tyler (1987), The atmosphere of Uranus: results of radio occultation measurements with Voyager 2, J. Geophys. Res., 92, 14,987-15,001.

Nakajima, K., S. Takehiro, M. Ishiwatari, and Y.-Y. Hayashi (2000), Numerical modeling of Jupiter's moist convection layer, Geophys. Res. Lett., 27, 3129-3133.

Reid, R. C., Prausnitz, J. M., and Poling, B. E. (1987), The Properties of Gases and Liquids, 4th ed., McGraw-Hill Companies.

Sugiyama, K., M. Odaka, K. Kuramoto, and Y.-Y. Hayashi (2001), Thermodynamic calculation of the atmosphere of the Jovian Planets, Proceedings of the 34 th ISAS Lunar and Planetary symposium, 53-56.

Van Zeggeren, F., and S. H. Storey (1970), The Computation of Chemical Equilibria, Cambridge Univ. Press, London.

Weidenschilling, S. J. and J. S. Lewis (1973), Atmospheric and cloud structure of the Jovian planet, Icarus, 20, 465-476.

White W. B., S. M. Johnson, and G. B. Dantxig (1953), Chemical Equilibrium in Complex Mixture, J. Chem. Phys., 28, 751-755.

Wood, J.A. and A. Hashimoto (1993), Mineral equilibrium in fractionated nebular systems, Geochimica et Cosmochimica Acta, 57, 2377-2388

K. Sugiyama, M. Odaka, K. Kuramoto, and Y.-Y. Hayashi, Division of Earth and Planetary Sciences, Hokkaido University, Sapporo 060-0810, Japan. (sugiyama@gfd-dennou.org) 